\title{
Computer holography becoming practical
}

\author{
M. Makowski \\ Faculty of Physics, Warsaw University of Technology, Koszykowa 75, 00-662 Warszawa
}

Received September 29, 2014; accepted September 29, 2014; published September 30, 2014

\begin{abstract}
This editorial contains the overview of the contents of the latest volume of the Photonics Letters of Poland, devoted mainly to holography. The published papers from international research groups show a slow but constant approach of holography towards practical applications in the future. This includes both scientific measurement techniques and future consumer electronics display devices.
\end{abstract}

This issue of the Photonics Letters of Poland is mainly devoted to holography. This area of optics is now intensively researched in many groups around the world, due to the availability of high resolution light modulators and extremely fast computing units. In this way holography follows the path towards practical applications in future devices, both in science and in consumer electronics.

In the paper by Makowski et al. there is presented a study of optimal resolution of a calculation matrix in holographic image projection. An increase in speed can be achieved by decreasing the mentioned resolution and without sacrificing any of the image contrast and noise ratio. Additionally, this allows better image resolution.

Kowalczyk et al. present the investigation on real-time computing of holograms for lensless image projection. They describe a successful and efficient implementation of the Iterative Fourier Transform Algorithm (IFTA) entirely on GPU processing. Real time processing was achieved for relatively large matrices and a successful experimental demonstration was achieved on a phaseonly spatial light modulator in color illumination.

Sugiyama, Ito et al. present the calculation results of computer digital holograms of ultra high resolutions up to $32 \mathrm{k} \times 32 \mathrm{k}$ points. The advantage of using solid-state drives compared to hard drives is assessed for many different sizes of the calculation matrix. In particular, the calculations with an SSD allowed obtaining a result after one-day computing, instead of one year as it was in the case of 40-billion-pixel holograms.

The Japanese group of Okada, Shimobaba et al. describes a new method of very efficient calculation (synthetising) of computer-generated holograms based on a wavefront recording plane. It assumes the representation of an input object with a bitmap containing an ordinary photograph and with the second bitmap containing a depth map of the object. This way of encoding 3-D information is more and more popular in modern stereoscopic cameras and TVs, therefore the presented calculation method is worth applying. Moreover, the presented calculations were performed on graphics processing units, which increased the speed by the factor of approx. one thousand compared to previously tested techniques.

Another paper dealing with holographic color display was given by Zaperty et al.. The authors show an experiment on a 3-D display with a spatial division of the SLM into color sections. A detailed study of the viewing geometry is given, followed by high quality pictures of the real experimental outcome.

Kakarenko et al. present a study of an optimal segmentation strategy of the surface of the SLM in order to obtain high quality projections with low (averaged) noise, high contrast and high resolution. In this detailed study they show the optimal display strategy with reasonable computational complexity.

An efficient and simple method of speckle noise averaging is presented in the paper by Ducin et al.. It is based on the sequential lateral shifting of a single Fouriertype hologram, which allows a fine reduction of speckle noise. This occurs with only one hologram, which makes this method extremely simple and fast. The author presents a study on optimal shifting strategy of a hologram for red, green and blue illumination.

Kostnecka et al. present an application of holography for the compensation of an error caused by significant defocus. This technique is suitable for improving the images reconstructed from tomographic phase microscopy by temporal phase shifting. Low computational complexity allows quick correction for efficient processing of multiple tomographic scans.

The study on the improvement of the second harmonic generation for future lasers and many other optoelectronic devices is described by Azeezaa et al.. The successful growth and characterization of a new highly non-linear crystal of L-cysteinium Sodium Nitrate Chloride is described with prospects of applications in photonics due to its promising dielectric properties.

The paper from an Iranian group by Noghani et al. provides the description of an interesting plasmonic directional coupler. Its extremely wide-band operation and compact size makes it a very promising medium for the next generations of splitters, combiners, switches, wavelength sorters etc. The authors have provided detailed characteristics of the proposed optical element.

Two papers of Ertman et al. and Chychłowski et al. present sophisticated techniques of chemical and photochemical bonding of special types of optical fibers. 